\title{
Voltage stabilization of distribution system integrated by renewable power generations by cooperated control of STATCOM and interconnecting microgrids
}

\author{
Hirotomo Miura, Guohong $\mathrm{Wu}^{*}$ \\ Department of Electrical Engineering \& Information Technology, the Faculty of Engineering, Tohoku Gakuin University. \\ 1-13-1, Chuo, Tagajyo, Miyagi-Prefecture, 985-8537, Japan
}

\begin{abstract}
This paper presents a method to improve the voltage stability of a distribution system that is integrated by renewable power generations and interconnected by microgrids, which can be expected to appear in the near future. Being impacted by the significant fluctuation of renewable powers, the distribution voltage may be considerably degraded along with the increasing integration of renewable power generations. However, even higher voltage quality is desirable in the modern society, and therefore, the voltage stabilization problem in the future distribution system comes to be a critical issue of concerns. In this paper, a new method is proposed to deal with this voltage stabilization problem by cooperated use of STATCOM (synchronous static compensator) and the reactive power control of interconnecting microgrids. With the proposed method, the reactive power from microgrids are controlled to mitigate the voltage change caused by the active power from these microgrids, and simultaneously, the STATCOM is actuated to suppress the voltage fluctuation originated from renewable power generators that are directly integrated into the distribution grid. In this paper, a STATCOM model of small capacity with relatively simple structure and low cost is proposed for distribution use, and the control systems for both the STATCOM and microgrids are designed as well. Digital simulations with a created typical future distribution system model are conducted in this study, and the simulations results have verified the voltage stabilization effects by the proposed method.
\end{abstract}

Keywords: Distribution system, microgrid, reactive power control, renewable power generations, STATCOM, voltage stabilization

\section{Introduction}

In the later years, with the increasing concerns of fossil energy resources exhaustion and global warming issues, the introduction of dispersed renewable power generations such as photovoltaic (PV) and wind generations is pushed forward rapidly. However, in spite of many advantages, some problems may be also caused in the power system management and control. These problems are mainly associated with the power fluctuation from renewable sources due to the essential characteristics of solar radiation and wind speed. And the distribution voltage may be considerably degraded following the increasing integration of renewable power generations in the near future, e.g. introduction of large amount of PV power generations into distribution systems like the case in Japan may possibly lead to voltage violation from the nominal value. For these reasons, the voltage stabilization problem with the distribution systems integrated by renewable power generations is considered to be an issue of great concerns and have to be solved appropriately. Up to now, some methods have been studied for distribution voltage enhancement. For the voltage rise and voltage fluctuations at a balanced condition, voltage control by SVR (step voltage regulator) or LRT (load ratio control transformer) are in practical use. However, it is considered that such distribution voltage controls are difficult to deal with the cases with diverse conditions of renewable

\footnotetext{
* Manuscript received May 24, 2013; revised July 29, 2013.

Corresponding author. Tel.: +81-022-368-7043; E-mail address: wugh@tjcc.tohoku-gakuin.ac.jp.
} 
power generations, and hardly respond to the voltage fluctuation of seconds' time order (e.g. response time of a SVR is normally at about 45-200 seconds). On the other hand, voltage controls by use of FACTS devices such as STATCOM (Synchronous Static Compensator) [1]-[5] and SVC (static var compensator) [6], [7] are considered to potentially obtain better voltage improving effect especially for the distribution systems integrated by large amount of renewable power generations. The work [1], [2] have shown the validly of STATCOM to enhance the voltage stability of a distribution network with wind power generations. In [3] and [4], the voltage fluctuation, imbalance and harmonics of a distribution system with PV power generations were effectively mitigated by a proposed STATCOM that is considered applicable to the distribution system. Paper [6] and [7] have discussed the effectiveness of SVC for enhancement of distribution voltage that is violated by PV power integrations. SVC is a reasonable choice in terms of cost, however there are also obvious disadvantages such as the necessity of large installation area, harmonics generation, degradation of voltage compensation effect following disturbances as well as its inability to compensate voltage imbalance. In [8], a microgeneration shedding method was employed and an optimization approach was developed for voltage control of distribution system. This method is thought to need a Distribution Control Center where to perform this operation.

For the future distribution system with increasing integration of renewable power generation, it is considered that introduction of STATCOM or SVC only may not be sufficient in terms of cost and efficient control, and the cooperated control of any available devices in the distribution system is therefore desirable. For this purpose, this paper proposes a method that involves in the cooperated reactive power control of both STATCOM and microgrids for voltage stabilization. Taking consideration of the facts that some of the renewable power generations will be interconnected to the grid directly and the others may be included into a microgrid and connects to the grid via a DC/AC inverter. Since the microgrid equipped with energy storages has the ability to manage and control its active power to the grid, the inverter for grid-connect hence has potential of efficient reactive power control for the purpose of voltage stabilization. In the proposed method, reactive power from microgrid/inverter is dispersedly controlled to mitigate the change of distribution voltage caused by its own active power penetration; and meanwhile, STATCOM is used to suppress the voltage fluctuation originated from those renewable power generators that are directly interconnected into the distribution grid. In this paper, a STATCOM model of small capacity and low cost is designed with a relatively simple structure, a microgrid model that consists of a DC bus and an AC bus interconnected by a DC/AC inverter is created, and a typical distribution system model is constructed taking consideration of large numbers of PV power generators that is estimated probably to appear in the near future in some areas like Japan. In addition, the cooperated control system of both the STATCOM and the microgrids are proposed for voltage stabilization. All of these models are completed by use of PSCAD/EMTDC (a simulation tools widely used in the field of power electronics) to conduct digital simulations in order to investigate the distribution voltage stabilization effects by the proposed method.

\section{STATCOM and Microgrid Model}

\subsection{Configuration of STATCOM}

STATCOM technologies for use in the high-voltage transmission system have already been well studied and presently in the step of practical use [9]. However, since STATCOM applied to distribution systems requires some essential properties such as low cost, simple structure and space-saving design, the configuration of STATCOM developed for high-voltage transmission system may be impractical for distribution use [10]. Taking consideration of this aspect, we have proposed a STATCOM for distribution system based on the existing technologies for STATCOM of large capacity and high-voltage. The proposed configuration of STATCOM is shown in Fig. 1 [3], [4], and it has the following properties:

- Less multiplex converter layers and using conventional transformer. Considering a STATCOM for distribution use requires only small capacity, converter layer is significantly reduced. Furthermore, a low cost conventional transformer is adopted. 
- Conventional voltage source converters using IGBT as power switching devices for PWM control.

- AC filters of small capacity.

The STATCOM operates to inject/absorb reactive power to/from the distribution system so as to control the voltage at the point where it is connected to the grid.

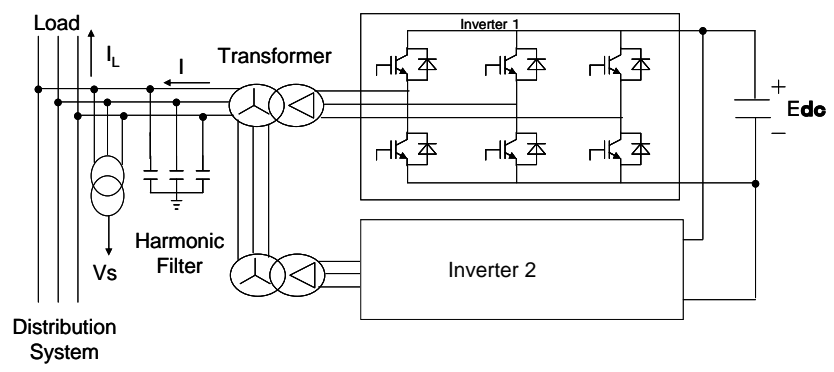

Fig. 1. Configuration of STATCOM.

\subsection{Configuration of microgrid}

Up to now, most of the renewable power generations are introduced in the way of directly connecting to the power grid, because this is the way with lower introduction cost. However, due to the uncertainty and fluctuation in renewable power sources, the power system management and control tend to be extremely complicated and difficult, and from point of view of the power system network management, the increase of direct integration of renewable power generation is undesirable. Thus, we can anticipate that in the future, beside the renewable power sources that have been already introduced, most of the renewable power sources will be required to add to the power grid as part of a microgrid, where the renewable power is first stabilized by the proper power control and storage of microgrid.

A microgrid is referred to as a small grid with an aggregation of loads and micro sources operating as a single system providing both power and heat. These micro sources may be some renewable sources such as wind power, solar power or some other cogenerations such as fuel cell, diesel turbine, gas turbine, etc. The macrogird normally has a function of energy management and power/voltage control itself, and may operate isolated from the power system or interconnected with the power system at one point. The configuration of microgrid model considered in this paper consists of a DC bus and an AC bus interconnected by a DC/AC inverter. The renewable power generators and battery are assumed to connect to the DC bus via power electronic-based circuits, and AC loads and power grid are connected to the AC bus. By proper control of the converted and stored power inside the microgrid, the DC bus voltage can be stabilized and the active power from this bus to AC bus can be controlled as well [11]. Therefore, the DC bus is assumed to be a P-controlled, constant DC voltage source in this work. In addition, DC/AC inverter is modeled in details by a typical 6-bridge 3-phases voltage source PWM inverter based on power electronics technologies, and reactive power control is applied to this inverter for voltage stabilization at the connection point.

\section{Control Systems}

The following voltage control strategy is though out in the proposed method:

(A) The distribution voltage may be deteriorated by two factors: 1) Fluctuating power penetrated from the renewable sources that are directly integrated to the distribution grid; 2) Active power flowing into the grid from the microgrids. And voltage is stabilized by mitigation of the impacts from both of these two factors, respectively.

(B) Voltage fluctuation originated from 1) is suppressed by a STATCOM.

(C) Voltage deterioration due to 2) caused by microgrid is mitigated by its own reactive power control.

\subsection{Control systems for STATCOM}

Control system for STATCOM designed in this work is shown in Fig. 2 [3]. It consists of two control 
blocks:

1) AC voltage regulation control

Mitigate the fluctuation and deviation in distribution voltage. AC voltage (r.m.s) is kept tracking its reference value by reactive power control employing the STATCOM.

2) DC voltage stabilization control

Maintain constant DC voltage in the capacitor bank. This control is necessary for continuous operation of the whole system, because the change of reactive power results in a change of capacitor voltage as well and it has to be compensated.
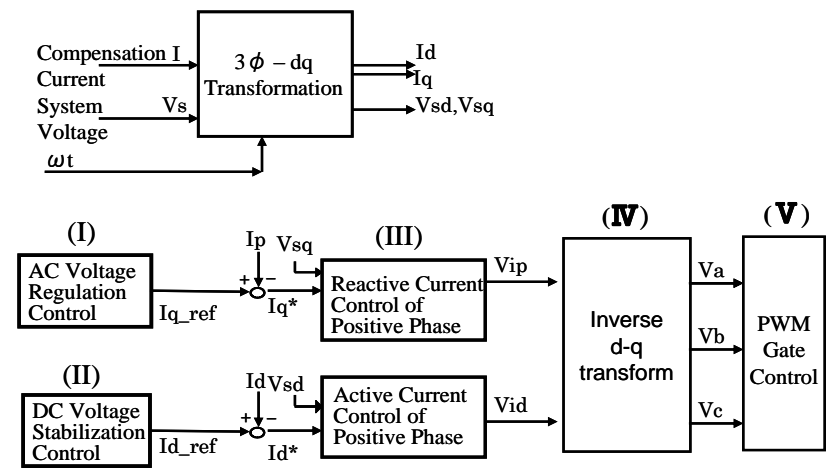

Fig. 2. Control system of STATCOM

\subsection{Control systems for microgrid}

Control system for microgrid inverter has the same main structure with that of the STATCOM, but the control purpose and control signals are different as described in the following.

1) DC bus voltage stabilization control

Mitigate the fluctuation and deviation in DC bus voltage of microgrids caused by renewable power generations. This is a coordinated control with that of the battery inside the microgrids. The active power that needs to be transferred to the grid by inverter is obtained by this control block and is referred to as $P_{i n}$.

2) AC Voltage regulation control

Control reactive power for voltage stabilization in the distribution system. In this control block, the deviation of distribution voltage is first calculated, and then, the reactive power reference $Q_{\text {ref }}$ is obtained via a PID control. And the actual Q reference is finally determined by a Reactive Power Maximum Output Control, which performs:

(a) The available reactive power capacity is calculated as

$Q_{i n_{-} \max }=\sqrt{S_{i n}^{2}-P_{i n}^{2}}$

where $S_{i n}$ is the rated capacity of inverter and $P_{i n}$ is the active power output.

(b) If $Q_{\text {in_max }}$ is larger than $Q_{\text {ref, }}$, then $Q_{\text {ref }}$ is set as the final $\mathrm{Q}$ reference; otherwise, if $Q_{\text {in_max }}$ is smaller than $Q_{\text {ref }}$, then $Q_{\text {in_max }}$ is set as the final Q reference.

\section{Simulation Conditions}

\subsection{Distribution system model}

Fig. 3 shows the distribution system model that is created for digital simulation studies. In this model, the distribution bus is assumed being connected by 6 feeders. Among them, 5 feeders are equivalently simulated by a distribution line and a load for simplification, and only one feeder is paid attentions and modeled in details. 12 residential houses are simplified to one load model, which is connecting to the nodes of \#1, \#2, \#4, \#5,\#7, \#8 and \#9, respectively. Among these residential houses, the ones at Node $\# 5$, \#7, \#8 and \#9 are assumed having introduced PV generation systems and directly connect to the grid. 
Besides, 3 microgrids with a capacity of $250 \mathrm{kVA}$ are integrated to Node \#3, \#6 and \#10, respectively. Parameters of transformers, transmission lines, loads and PV generations can be found in Fig. 3. STATCOM is installed at Node \#6 where is the tap-changing point and feasible to voltage deviation in this system.

Distributed PV generators are modeled by variable power sources considering MPPT (Maximum Power Point Trace) control. Solar power is calculated based on the measured solar radiation data obtained at Tagajo campus of Tohoku Gakuin University, Japan. All the PV generators are assumed to receive the same amount of solar radiation and give the same power generation, which is around $50 \mathrm{~kW}$ with a slow decrease to minimum $28 \mathrm{~kW}$ during 22 - $40 \mathrm{sec}$.

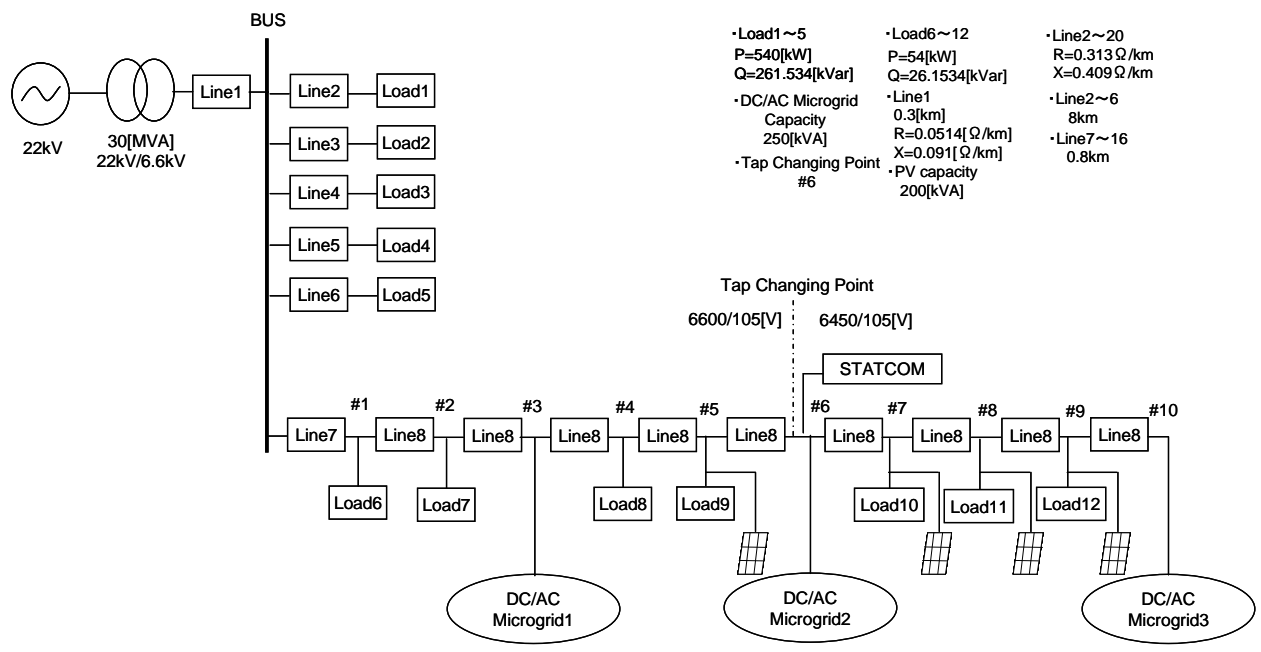

Fig. 3. Distribution system model.

Table 1. Specifications and parameters of STATCOM model

\begin{tabular}{|l|l||l|l||}
\hline Rated Capacity & $500 \mathrm{kVA}$ & Frequency & $50 \mathrm{~Hz}$ \\
\hline Rated AC Voltage & $6.6 \mathrm{kV}$ & Rated DC Voltage & $0.3375 \mathrm{kV}$ \\
\hline Rated AC Current & $0.0435 \mathrm{kA}$ & DC Capacitor & $12 \mathrm{mF}$ \\
\hline PWM Pulse & 100 & AC Harmonic Filter & $50 \mu \mathrm{F}$ \\
\hline Transformer & $Y-4$ & Power Device & IGBT \\
\hline
\end{tabular}

\subsection{Specifications and parameters for STATCOM}

Table 1 gives the specifications of STATCOM used in this simulation study. Capacity of STATCOM is assumed to be $500 \mathrm{kvar}$, which is determined to satisfy the requirement for mitigation of the rise in distribution voltage to the criteria level of $101 \pm 6 \mathrm{~V}$.

\subsection{Operating conditions of microgrids}

Taking consideration of the power from renewable sources included in the microgrids can be controlled by use of energy storage devices, the active power from microgrid to the grid is assumed to be stable and changeable. In this simulation, it is set as

$150 \mathrm{~kW}$ (during 0-30 sec.) $\rightarrow 180 \mathrm{~kW}$ (during 30-50 sec.) $\rightarrow 130 \mathrm{~kW}$ (during 50-60 sec.)

\section{Simulation Results}

Digital simulation studies are conducted in order to confirm the voltage stabilization effect by the cooperated control of STATCOM and microgrids. Two issues are to be verified in this study:

1) Whether the cooperated control can efficiently stabilize the voltage to satisfy the criterion.

2) Whether there are interference occurring between the control of STATCOM and microgrid, which may bring about some unexpected facts. 


\subsection{Voltage of distribution system}

For verification of the voltage stabilization effect, the comparison between the voltage curves at the major nodes in case with and without reactive power controls is shown in Fig. 4. Fig. 4 (a) is that in case without cooperated control, and that in case with control is given in Fig. 4 (b). From Fig. 4, it is known that in case without control, the maximum voltage (r.m.s) at Node \#6 and \#10 are obviously beyond the acceptable value of $101 \pm 6 \mathrm{~V}$. On the other hand, in case with the cooperated control, both of the voltages at these nodes are improved to a satisfied level and the deviation of voltage profiles are improved effectively as well. These results have illustrated the validity of the proposed method for dealing with the voltage degradation caused by PV integrations in this case.

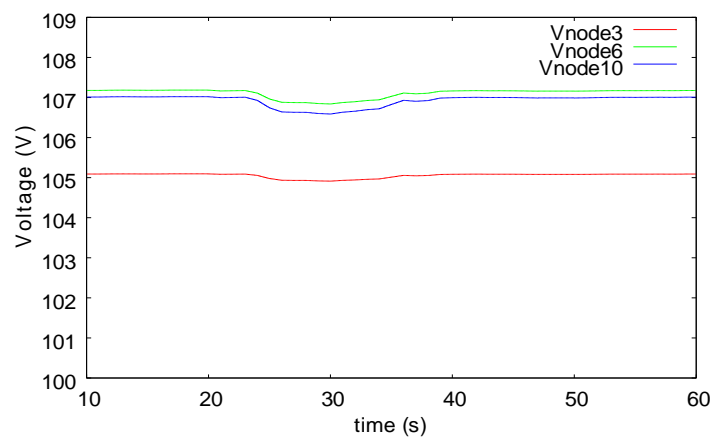

(a) Voltage curve in case without controls

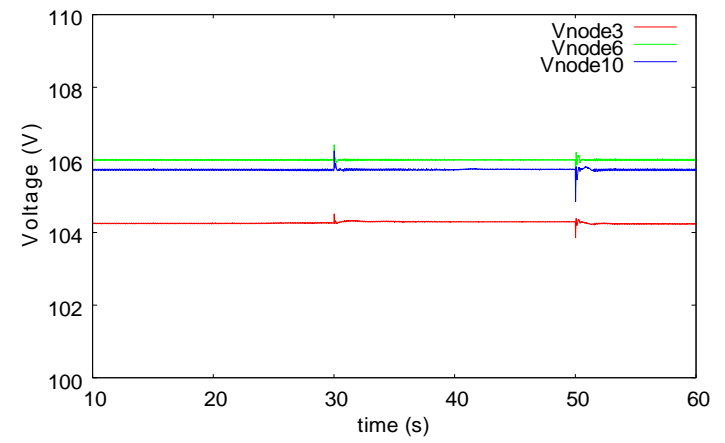

(b) Voltage curve in case with controls

Fig. 4. Simulation results of voltage at Node \#3, \#6 and \#10.

\subsection{Reactive power output}

Reactive power results from STATCOM and microgrids are shown in Fig. 5 and Fig. 6, respectively.

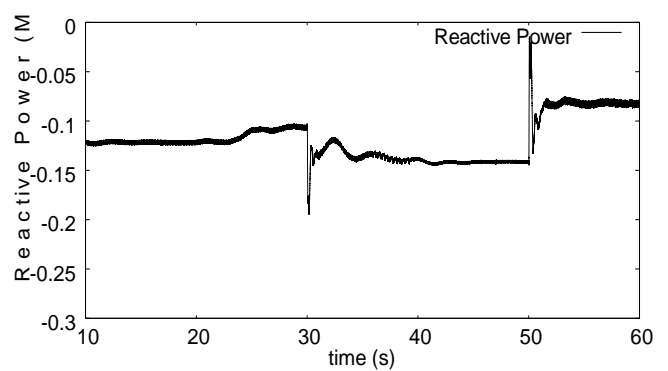

Fig. 5. Reactive power of STATCOM.

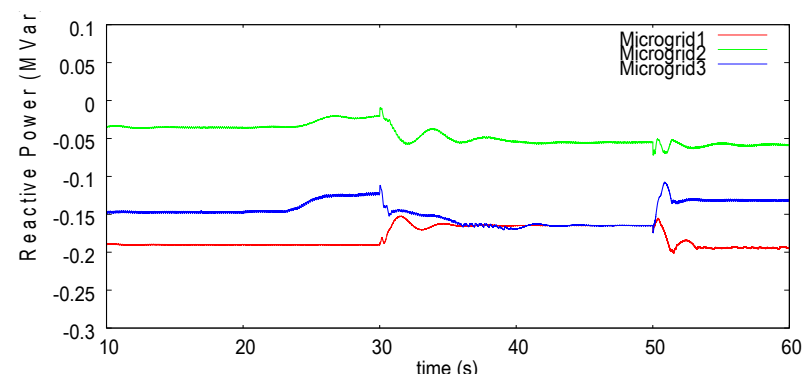

Fig. 6. Reactive power of STATCOM of microgrids.

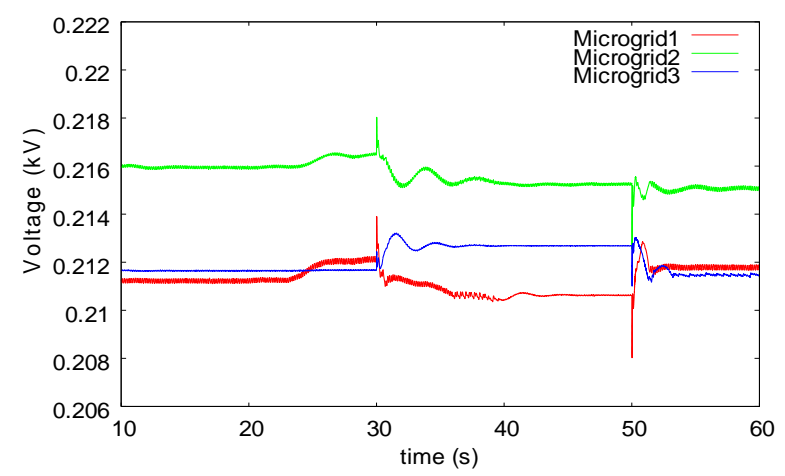

Fig. 7. AC voltage curve of microgrids. 
From these figures, it can be seen that both the STATCOM and the mocrogrids is absorbing reactive power so as to reduce the rise in voltage that is caused by PV power integrations and active power from microgrids, and these reactive powers are varying in response to the change of PV generations. Furthermore, it is noticed that during the time of 30-50 sec. while the active power from all the microgrids are increased, the active power that need to be delivered from the distribution station is therefore reduced and the voltage at Node \#6 tend to arise. Simultaneously, STATCOM increase its reactive power absorption so as to keep it constant, while microgrid 2 at the same node also increases its reactive power as a support. At this time, if considering the voltage before and after $30 \mathrm{sec}$., it can be estimated that the voltage at bus \#10 will increase while that at bus \#3 will decrease, due to the reduction of the active power flowing on the distribution line. This process has been confirmed by observing the AC voltage curves of each microgrid given in Fig. 7. And in response to the voltage change, microgrid 1 at Node \#3 reduces its absorbed reactive power and microgrid 3 at Node \#10 increases it reversely.

In addition, transient phenomena especially following the operation of STATCOM are observed with these curves. These are considered that could be improved by optimization of control systems.

\subsection{AC voltage of microgrids}

Fig. 7 shows the simulation results of AC voltage inside the micrograms. It can be confirmed from this results that although the $\mathrm{AC}$ voltage is lightly fluctuating due to the impact of its active power change, the voltage values of all these microgrids are controlled properly to be within the criterion of $202 \pm 20 \mathrm{~V}$. And during the time of 30-50 sec. with active power changes from these microgrids, although the AC voltage of the microgrids at different node shows different changing pattern, however, all these microgrids can properly control their reactive power to stabilize the voltage.

\section{Conclusions}

This paper has presented a method to deal with the voltage deterioration problem in a distribution system integrated by renewable power generations. The proposed method has considered the cooperated reactive power control of a STATCOM and the microgrids interconnecting to the grid.

In this work, a STATCOM model with lower cost and simple structure is designed and thought to be suitable for distribution use, and the control systems for both the STATCOM and microgrids are proposed for stabilization of distribution voltage. The fluctuation of distribution voltage is cooperatively mitigated by dispersed reactive power control of STATCOM and microgrid using local control signals. Based on the renewable power introduction targets indicated by the government, a distribution system model including PV generations with proper introduction rate and typical microgrids is created, and digital simulations have been conducted to verify the effectiveness of the proposed voltage stabilization method.

As a result, it is verified in this work that by properly cooperated reactive power control of STATCOM and microgrid inverter, both the voltage of distribution system and that of the microgrids can be stably suppressed to an appropriate value.

\section{Acknowledgements}

Herby, we would like to thank to Mr. Yuma Takada and Kazutoshi Chubachi, who are now working with Tohoku Electric Co. LTD., Japan, for their great contributions to this work.

\section{References}

[1] Sharaf AM, Wang W, Atlas IH. Novel STATCOM controller for reactive power compensation in distribution networks with dispersed renewable wind generation. In: Proc. of Canadian Conference on Electrical and Computer Engineering, 2007:15821585.

[2] Han C, Huang AQ, Baran M et al. STATCOM impact study on the integration of a large wind farm into a weak loop power system. IEEE Trans. Energy Conversion, 2008; 23(1):226 - 233.

[3] Takada Y, Wu G. A study of quality enhancement by introduction of STATCOM in distribution system with dispersed 
generations. In: Proc. Int. Conf. on Electrical Engineering, Kanazawa, Japan, 2012:876-881.

[4] Wu G, Chubachi K, Huang CH, Kang L. An approach to voltage quality improvement by introduction of STATCOM for distribution system with renewable power generations. In: Proc. IEEE PES Innovative Smart Grid Technologies Asia, Tianjin, China, 2012:1-6.

[5] Qiao W, Harley RG, Venayagamoorthy GK. Coordinated reactive power control of a large wind farm and a STATCOM using heuristic dynamic programming. IEEE Trans. Energy Conversion, 2009; 24(2):493-503.

[6] Iioka D, Sakakibara K, Yokomizu Y, Matsumara T, Norihisa M. Distribution voltage rise at dense photovoltaic power generation area and its suppression by SVC. IEEJ Trans. PE, 2006; 126(2):153-158 (in Japanese).

[7] Kondo T, Baba J, Yokoyama A. Voltage control of distribution network with a large penetration of photovoltaic generations using FACTS Devices. IEEJ Trans. PE, 2006; 126(3):347-358 (in Japanese).

[8] Madureira AG, Peças Lopes JA. Coordinated voltage support in distribution networks with distributed generation and microgrids. Journal of IET Renewable Power Generation, 2009; 3(4):439-454.

[9] Fuji T, Funabashi S, Morishima N et al. A \pm 80 MVA GCT STATCOM for the Kanzaki substation. In: Proc. IPEC-Nigata, 2005:1299-1306.

[10] The Power to Change - Stabilizing grids and enabling renewable power generation with PCS 6000 STATCOM. ABB Review Jan. 2010:16-19.

[11] Wu G, Kodama S, Ono Y, Monma Y. A hybrid microgrid system including renewable power generations and energy storages for supplying both the DC and AC loads. In Proc. Int. Conf. Renewable Energy Research and Applications (ICRERA2012), Nagasaki, Japan, 2012:1-5. 